\title{
Does mechanical ventilation precipitate gastro-oesophageal reflux during enteral feeding?
}

\author{
S J NEWELL, ${ }^{*}$ M E I MORGAN, $\dagger$ G M DURBIN, $\dagger$ I W BOOTH, ${ }^{*}$ AND A S McNEISH* \\ ${ }^{*}$ Institute of Child Health, University of Birmingham and $\nmid$ Regional Neonatal Unit, Birmingham Maternity \\ Hospital
}

SUMMARY The influence of intermittent positive pressure ventilation on gastro-oesophageal reflux in preterm infants is not known. In many neonatal units, however, concern that ventilation may increase gastro-oesophageal reflux (and therefore aspiration) leads to avoidance of enteral feeding during ventilation. We have therefore performed a crossover study of gastrooesophageal reflux by monitoring lower oesophageal $\mathrm{pH}$ in a group of nine enterally fed, very low birthweight infants both during assisted ventilation and normal breathing. All infants had less reflux during intermittent positive pressure ventilation (mean (SEM) reflux index $2 \cdot 3(0.6 \%)$ ) than during normal breathing (mean (SEM) reflux index $6 \cdot 1(1 \cdot 1 \%)$ ). Assisted ventilation was associated with a significant reduction in the gastro-oesophageal pressure gradient, an effect which may be related to the use of positive and end expiratory pressure during ventilation. These data show that fear of gastro-oesophageal reflux should not preclude the use of enteral feeding in preterm infants receiving ventilation.

Early and successful enteral nutrition of preterm infants has many advantages, including reduced reliance on parenteral nutrition ${ }^{1}$ and the avoidance of its complications, ${ }^{2}$ the emotional reward for mothers who provide milk for their babies, and the possibility of less osteopenia. ${ }^{1}$ Infants who receive even small volumes of milk also show improved tolerance of feeds, reflecting a likely acceleration of gut maturation, ${ }^{3}$ which may be mediated through the postcibal release of enteric hormones. ${ }^{4}$ There are, however, possible disadvantages. During intermittent positive pressure ventilation (IPPV) preterm infants are said to be at high risk of gastrooesophageal reflux, perhaps due to uncoordinated oesophageal peristalsis. ${ }^{5}$ Regurgitated milk, once present in the oropharynx, may then be aspirated around a non-cuffed endotracheal tube. ${ }^{6}$

The association between enteral feeding, IPPV, and gastro-oesophageal reflux, however, has not previously been studied. This scarcity of evidence about the use of milk feeds during IPPV has led to wide variations in feeding policies. In a telephone survey of regional neonatal intensive care units we found that although in most units milk feeds were routinely introduced early during ventilation, in $36 \%$ of units milk feeds were seldom used during
IPPV, and then only if long term ventilation was anticipated.

We have therefore performed a crossover study designed to determine the effect of IPPV upon gastro-oesophageal reflux in very low birthweight infants receiving intragastric feeds.

\section{Subjects and methods}

Nine infants were studied, all of whom were preterm (mean (SEM) gestation $27(0.4)$ weeks), and of very low birth weight $(1.04(0.07) \mathrm{kg})$. During the study they were receiving at least $90 \mathrm{ml} / \mathrm{kg} / \mathrm{day}$ of expressed breast milk as hourly bolus feeds through nasogastric tubes. Infants were closely observed by the nursing staff for signs of abdominal distension. The nasogastric tubes were aspirated four hourly before feeds, and feeds were only continued if the residual volume did not exceed $3 \mathrm{ml}$; most residual volumes were less than $1 \mathrm{ml}$.

Each infant was studied twice. In eight infants the first study was during IPPV, and the second while breathing spontaneously. The first study was performed at a mean (SEM) postconceptional age of 29 $(0.5)$ weeks while receiving $123(8) \mathrm{ml} / \mathrm{kg} /$ day of milk feed, and the second during normal breathing 
at a mean postconceptional age of $30(0 \cdot 3)$ weeks, while receiving $142(8) \mathrm{ml} / \mathrm{kg} /$ day of milk feed. The differences in feed volumes were not significant.

The primary respiratory illnesses requiring IPPV were hyaline membrane disease $(n=4)$, chest infection $(n=2)$, congenital pneumonia $(n=1)$, and chronic lung disease $(n=2)$. Infants were ventilated on a time cycled, pressure limited ventilator, either the Drager Babylog (8403/300), or the Sechrist infant ventilator (100B). Positive end expiratory pressure of 3-5 $\mathrm{cm} \mathrm{H}_{2} \mathrm{O}$ was used for all infants on IPPV. During the study none of the infants was receiving sedation, muscle relaxants, or xanthine derivatives.

During the study infants continued to receive routine nursing care, which included chest physiotherapy for a few minutes carried out by a member of the nursing staff while the infant remained in the incubator. After physiotherapy mucus was aspirated from the oropharynx and endotracheal tube with a fine catheter. When necessary the nappy was changed in the incubator and a clean nappy loosely applied. There was no significant difference between the mean (SEM) number of episodes of nursing care during IPPV (13.1 (2.9) episodes/24 hours) and normal breathing $8.9(2.2)$ episodes/24 hours. Infants were nursed in the prone, right lateral, and left lateral positions, and spent equal times in each position during both studies.

The protocol was approved by the district research ethics committee, and informed consent was obtained from the parents before each study.

A $1 \mathrm{~mm}$ monocrystalline antimony electrode (Monocrystant-infant, Synectics Medical) was used to monitor lower oesophageal $\mathrm{pH}$ continuously for 24 hours as previously described. ${ }^{7}$ The $\mathrm{pH}$ electrode was bonded to a nasogastric tube (external diameter $1.2 \mathrm{~mm}$ ) attached to a continuously perfused manometric system; this was used to identify the lower oesophageal sphincter and thereby position the nasogastric tube in the stomach with the $\mathrm{pH}$ electrode in the lower oesophagus $1-2 \mathrm{~cm}$ above the lower oesophageal sphincter. During the study infants were fed through the nasogastric tube and no change was made in their routine care.

The manometric system was used at the end of each study to measure resting lower oesophageal sphincter pressure as previously described. ${ }^{8}$ Pressure measurements were made in eight of the infants during IPPV, and in all nine infants during normal breathing. The gastro-oesophageal pressure gradient (that is, the difference between the resting end expiratory pressure in the fundus of the stomach and in the body of the oesophagus) was then calculated.

Twenty four hour $\mathrm{pH}$ recordings were analysed, and the following variables were recorded: the number of episodes/24 hours; the number of episodes lasting longer than 5 minutes in every 24 hours; the reflux index $\%$ (the percentage of the total observation period during which the $\mathrm{pH}$ was less than 4 ) and the longest single episode (in minutes) in 24 hours. The reflux index was used as the simplest overall measure of the amount of reflux. ${ }^{9}$

Statistical analyses were performed by the paired Student's $t$ test, and Wilcoxon's rank sum test. All means are shown with standard errors and, where comparisons are made, with the standard error of the mean of the differences (SE diff):

\section{Results}

Throughout the study the nine infants tolerated feeds well and there was no clinical evidence of gastrointestinal problems. In particular there was no vomiting and at no time was milk aspirated from the oropharynx.

During normal breathing there was significantly more gastro-oesophageal reflux than during ventilation, with an almost threefold increase in the reflux index from $2 \cdot 3(0 \cdot 6)$ to $6 \cdot 1(1 \cdot 1) \%$ (SE diff $0 \cdot 8$,

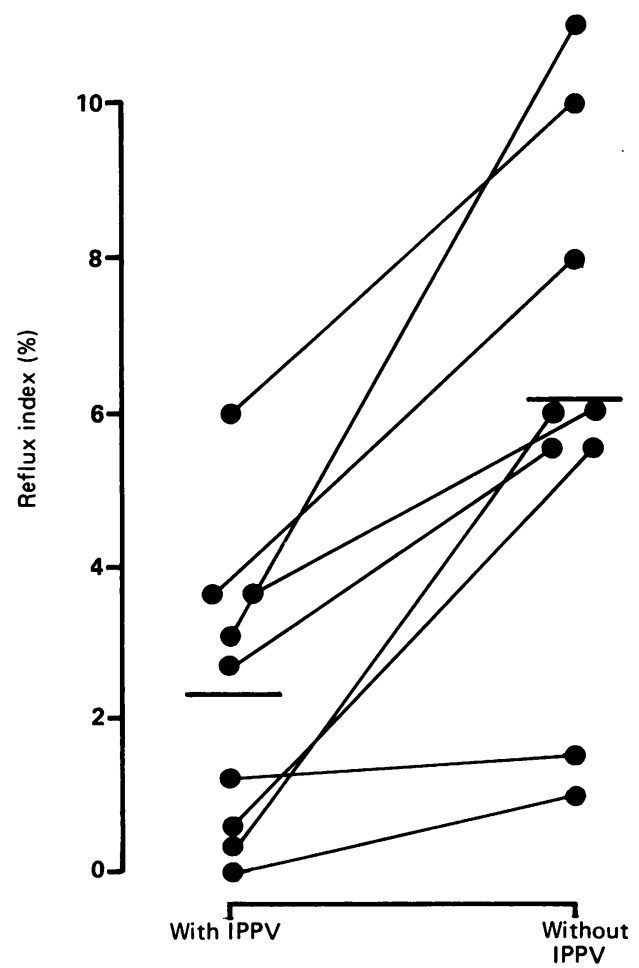

Fig 1 Reflux index (\%) in nine infants during assisted ventilation (with IPPV) and normal breathing (without IPPV). Means are shown as solid bars $(p<0 \cdot 01)$. 
Table Gastro-oesophageal reflux and lower oesophageal sphincter pressures in nine infants during ventilation (IPPV) and normal breathing. Values are shown as mean (SEM)

\begin{tabular}{|c|c|c|c|}
\hline & $\begin{array}{l}\text { Normal } \\
\text { breathing }\end{array}$ & $I P P V$ & $p$ Value \\
\hline $\begin{array}{l}\text { No of episodes in } 24 \text { hours } \\
\text { No of episodes lasting } \\
\text { longer than }\end{array}$ & $19.4(3.4)$ & $12 \cdot 2(2 \cdot 6)$ & $>0.05$ \\
\hline $5 \mathrm{mins} / 24$ hours & $5 \cdot 1(1 \cdot 2)$ & $2.2(0.9)$ & $<0.05$ \\
\hline Longest episode (mins) & $14 \cdot 7(3 \cdot 1)$ & $7 \cdot 2(2.0)$ & $<0.02$ \\
\hline Reflux index (\%) & $6 \cdot 1(1 \cdot 1)$ & $2 \cdot 3(0 \cdot 6)$ & $<0.01$ \\
\hline $\begin{array}{l}\text { Resting lower oesophageal } \\
\text { sphincter pressure } \\
\text { (mm Hg) }\end{array}$ & $8.4(0.5)$ & $7.0(1.0)$ & $>0.05$ \\
\hline
\end{tabular}

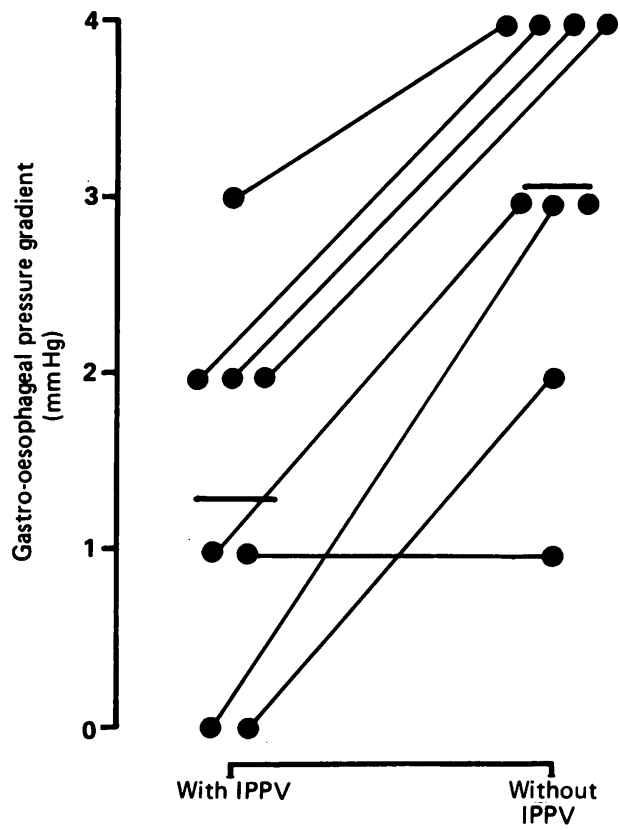

Fig 2 Gastro-oesophageal pressure gradients in eight infants during assisted ventilation (with IPPV) and nine infants during normal breathing (without IPPV). $(p=0.01$, Wilcoxon's rank sum test $)$.

$\mathrm{p}<0.01$ ) (fig 1). There were significant rises in the number of prolonged episodes (SE diff 1.3, $p<0.05$ ), and the mean longest episode (SE diff $2 \cdot 3, \mathrm{p}<0 \cdot 02$ ), although the increase in the total number of episodes did not achieve significance (table).

Oesophageal manometry showed no difference in mean lower oesophageal sphincter pressure between studies carried out during IPPV and normal breathing. The gastro-oesophageal pressure gradient during normal breathing, however (3.1 mm $\mathrm{Hg}$, range $1-4)$, was significantly increased compared with that during IPPV $(1 \cdot 4$, range $0-3, p=0 \cdot 01)$ (fig 2$)$.

\section{Discussion}

In this crossover study of gastro-oesophageal reflux in infants receiving intermittent intragastric feeds, three times more gastro-oesophageal reflux and an increase in the gastro-oesophageal pressure gradients were observed during normal breathing compared with during assisted ventilation.

An early trial of feeding practice conducted in 1965 compared preterm infants on a regimen of early introduction of rapidly increasing volumes of milk feeds with those who had later introduction of smaller volumes of feed. A higher mortality was found in those on higher volumes of milk; necropsy studies suggested that this was caused by aspiration pneumonia. ${ }^{10}$ Enteral feeds are still avoided in some units because of the fear of gastro-oesophageal reflux and subsequent pulmonary aspiration. ${ }^{5611}$ Indeed, in a recent textbook of neonatology it is stated that the early introduction of oral or nasogastric feeds in babies with respiratory distress syndrome is one of the commonest errors of management. ${ }^{5}$ It is also stated, however, that the cautious early introduction of milk feeds in preterm infants not being ventilated is now common practice, ${ }^{5}$ and we have found that in most regional neonatal intensive care units enteral feeds are now used during ventilation. In these units milk is introduced to avoid parenteral nutrition if possible, and with putative beneficial effects upon gut maturation ${ }^{12}$ and motility, ${ }^{3}$ and tolerance of feeds. ${ }^{1}$

Clearly the wide variation in attitude towards the use of enteral milk feeds among regional neonatal centres in the United Kingdom is compatible with the lack of compelling evidence for or against the safety of enteral feeding in preterm infants receiving IPPV.

We were surprised to find less gastro-oesophageal reflux during ventilation. During normal breathing the reflux index did not differ from our previously reported overall mean for the preterm, intensive care population. ${ }^{7}$ Although we have previously shown that gastro-oesophageal reflux is increased after feeds and nursing care, there were no significant differences between these variables in the two study periods to explain the observed differences in gastro-oesophageal reflux. In fact, during IPPV the number of episodes of nursing care was marginally higher and endotracheal suction was also carried out, both of which might be expected to increase, rather than decrease, the amount of gastro-oesophageal reflux. Furthermore, the observed fall in the reflux index during ventilation was mainly the 
result of a reduction in the number of prolonged episodes of reflux. It is possible, therefore, that during IPPV either oesophageal clearance of gastric contents is improved, or that there is a reduction in the volume of gastro-oesophageal reflux.

The use of positive end expiratory pressure may be an important factor in the reduction of gastrooesophageal reflux seen during IPPV. Positive end expiratory pressure results in positive intrathoracic pressure throughout the respiratory cycle and alters the physiological pressure difference between the abdominal and thoracic cavities. During normal breathing the resting pressure in the stomach exceeds that in the body of the oesophagus, thereby providing a positive gastro-oesophageal pressure gradient that predisposes towards reflux. ${ }^{13}$ Constant reflux is prevented by, among other factors, the presence of the lower oesophageal sphincter. In this study the gastro-oesophageal pressure gradient during normal breathing was similar to that previously reported by us. ${ }^{8}$ During IPPV, however, which included the use of positive end expiratory pressure, the gastro-oesophageal pressure gradient was significantly reduced $(p=0.01)$ (fig 2$)$. The reduction in this pressure gradient, which predisposes to gastro-oesophageal reflux, may therefore help to explain our findings. It is noteworthy that we did not find any difference in resting lower oesophageal sphincter pressure between periods of IPPV and normal breathing.

Advice and practice for the optimal nutrition of preterm infants vary greatly, and it is clear that this area of clinical practice requires further critical evaluation. The data presented provide further evidence that enteral nutrition is not contraindicated during IPPV.

Simon Newell was sponsored by the National Fund for Research into Crippling Diseases (Action Research) and gratefully acknowledges their generous support. We thank Synectics Medical who provided the antimony $\mathrm{pH}$ electrodes, and $\mathrm{Mr} \mathrm{GW}$ Hollins, $\mathrm{Mr} \mathrm{PH}$ Williams, and Mr T Jones of the medical physics department of the Birmingham Maternity Hospital for their technical expertise, the medical and nursing staff of the neonatal unit for their cooperation, and the participants in the questionnaires.

\section{References}

' Dunn L, Hulman S, Weiner J, Kliegman R. Beneficial effects of early hypocaloric enteral feeding on neonatal gastrointestinal function: preliminary report of a randomized trial. $J$ Pediatr 1988;112:622-9.

2 Postuma R, Terence CL. Liver disease in infants receiving total parenteral nutrition. Pediatrics 1979;63:431-4.

3 Bisset WM, Watt J, Rivers R, Milla P. The ontogeny of small intestinal motor activity. Pediatr Res 1986;20:692.

${ }^{4}$ Lucas A, Bloom SR, Aynsley Green A. The development of gut hormone response to feeding in neonates. Arch Dis Child 1980;55:678-82.

$\checkmark$ Roberton NRC. Pulmonary disease in the newborn. In: Roberton NRC. Text book of neonatology. Edinburgh: Churchill Livingstone, 1986: 259-311.

6 Goodwin SR, Graves SA, Haberkern CM. Aspiration in the intubated premature infant. Pediatrics 1985;75:85-8.

7 Newell SJ, Morgan MEI, Durbin GM, Booth I'W, McNeish AS. Gastro-oesophageal reflux in the preterm infant. Arch Dis Child 1989;64:780-6.

${ }^{8}$ Newell SJ, Sarkar PK, Durbin GM, Booth IW, McNeish AS. Maturation of the lower oesophageal sphincter in the pre-term baby. Gut 1988;127:167-72.

${ }^{9}$ Vandenplas Y, Sacre L. Continuous 24-hour oesophageal pH monitoring in 285 asymptomatic infants 0.15 months old. $J$ Pediatr Gastroenterol Nutr 1987;6:220-4.

10 Wharton BA, Bower BD. Immediate or later feeding for premature babies? A controlled trial. Lancet 1965;ii:969-72.

1 Nussbaum E, Maggi JC, Mathis R, Galant SP. Association of lipid-laden alveolar macrophages and gastroeosophageal reflux in children. $J$ Pediatr 1987;110:190-4.

12 Mayne AJ, Brown GA, Sule D, McNeish AS. The post-natal development of dissacharidase activities in the jejunal fluid of pre-term neonates. Gut 1986;27:1357-61.

13 Cohen S, Harris LD. The lower oesophageal sphincter. Gastroenterology 1972;63:1066-73.

Correspondence to Dr SJ Newell, Institute of Child Health, The Nuffield Building, Francis Road, Birmingham B16 8ET.

Accepted 6 April 1989 